\title{
Intracellular Chloride Ion Channel Protein-1 Expression in Clear Cell Renal Cell Carcinoma
}

\author{
ALEXANDRU NESIU ${ }^{1}$, ANCA MARIA CIMPEAN ${ }^{2,3}$, RALUCA AMALIA CEAUSU $^{2,3}$, AHMED ADILE $^{1}$, \\ IOAN IOIART ${ }^{1}$, CAMILLO PORTA $^{4}$, MICHELE MAZZANTI $^{5}$, TOMMASO CIRO CAMEROTA $^{6}$ and MARIUS RAICA ${ }^{2,3}$ \\ ${ }^{1}$ Department of Urology, Vasile Goldis University, Arad, Romania; \\ ${ }^{2}$ Department of Microscopic Morphology/Histology, \\ Victor Babes University of Medicine and Pharmacy, Timisoara, Romania; \\ ${ }^{3}$ Angiogenesis Research Center, Victor Babes University of Medicine and Pharmacy, Timisoara, Romania; \\ ${ }^{4}$ Department of Internal Medicine, University of Pavia \& Division of Translational Oncology, \\ IRCCS ICS Maugeri of Pavia, Pavia, Italy; \\ ${ }^{5}$ Department of Biosciences, Laboratory of Cellular and Molecular Physiology, University of Milano, Milan, Italy; \\ ${ }^{6}$ Urology, IRCCS ICS Maugeri of Pavia, Pavia, Italy
}

\begin{abstract}
Background/Aim: Chloride intracellular channel 1 (CLIC1) represents a promising target for personalized therapy. Our aim was to assess CLIC1 expression in clear cell renal cell carcinoma (cc RCC) and identify its possible prognostic role. Materials and Methods: Fifty cases of cc RCC were evaluated and selected for immunohistochemistry. CLICI expression was correlated with tumor grade, invasion and heterogeneity. Results: A total of $87.5 \%$ of the cases were CLIC1 positive, with either a homogeneous $(31.42 \%)$ or a heterogeneous $(68.57 \%)$ pattern. Low, mild and strong CLIC1 expressing tumors were defined based on nuclear $(N)$, cytoplasmic $(C)$, membrane $(M)$ or combinations of them (NC, NM, CM, NCM) in terms of CLIC1 distribution. A significant correlation was found between tumor grade and percent of positive tumor cells $(p=0.017)$. For G3 tumors, CLIC1 cytoplasmic expression was strongly correlated with high expression status $(p=0.025)$ and tumor heterogeneity ( $p=0.004)$. CLIC1 expression was also correlated with metastasis ( $p=0.046)$. Conclusion: We defined four cc RCC groups depending on G, CLIC1 expression and pattern: i) G3/NM/low CLICl ${ }^{+}$, ii) G2/CM/mild CLICl ${ }^{+}$iii) G1 or G2/NM or CM /high CLICl ${ }^{+}$, and iv) G2/M /high CLICl ${ }^{+}$.
\end{abstract}

This article is freely accessible online.

Correspondence to: Anca Maria Cimpean, MD, Ph.D., Hab. Dr, Professor "Victor Babes" University of Medicine and Pharmacy, Department of Histology, Piata Eftimie Murgu nr. 2, 300041 Timisoara, Timis, Romania. Tel: +40 720060955, e-mail: ancacimpean1972@ yahoo.com

Key Words: Chloride Intracellular Channel 1 (CLIC1), clear cell renal cell carcinoma (cc RCC).
CLIC1 (chloride intracellular channel 1) is a protein that belongs to the family of ion channels of chlorine. This protein is naturally expressed in the human body and is involved in many cellular processes, such as cell volume regulation, regulation of membrane potential, cell cycle regulation, cell proliferation and cell differentiation (1). CLIC1 normally exists as a soluble globular protein, however, in response to oxidative stress, it translocates from the cytoplasm to the cell membrane where it selectively acts as a chloride channel $(2,3)$. A potential involvement of CLIC1 in tumor development is suspected both for its role in cell cycle regulation as well as for its functional expression during oxidative stress.

Amongst the six members of intracellular chloride ion channel protein family (CLICs), CLIC1 and CLIC4 have been extensively studied regarding their involvement in tumor development (4). Indeed, CLIC1 is overexpressed in several tumor types, such as gastric cancer (5), oral squamous cell carcinomas (6) or glioblastoma (7). Recently, Barbieri et al. reported that CLIC1 inhibition induces glioblastoma growth inhibition, mainly by acting on CLIC1-rich glioblastoma stem cells, decreasing their proliferation and subsequently tumor progression (8). Although CLIC1 expression in cancer stem cells (CSC) has been certified only for glioblastoma $(7,8)$, indirect evidence suggests that CLIC1 is also expressed in tumor stem cells from other aggressive malignancies, such as pancreatic (9), ovarian (10) and esophageal cancer (11).

CLIC1 is characterized by a high versatility regarding its ability to translocate from cytosol to the nucleus and/or the plasma membrane during malignant transformation (12), based on its ability to spontaneously convert from soluble to an integral membrane-bound form. This changeable state is most probably regulated by the membrane lipid composition, especially by cholesterol, together with external factors, such 
as oxidation and $\mathrm{pH}(13,14)$, however, a precise mechanism is currently unknown. Once CLIC1 is incorporated in the plasma membrane, tumor cells become highly active, with a rapid proliferation rate (15), increased invasiveness (16) and metastatic potential (17).

Kidney cancer accounts for 5\% and 3\% of all malignanciens in adult men and women, respectively (18). The most frequent histological type (around 80\%) is represented by clear cell renal cell carcinoma (cc RCC). Cc $\mathrm{RCC}$ is a kidney malignancy with an unpredictable behaviour, especially in terms of response to treatment (19). Although in most cases kidney cancer is diagnosed when already localized, approximately $20 \%$ of patients will develop metastasis following nephrectomy with curative intent (20). In other cases, cc RCC is already invasive at the time of diagnosis and, despite of new targeted therapies, patients present a high mortality rate (16). This suggests that the spectrum of cc RCC is not fully characterized regarding its molecular profile. Unpredictable response to treatment represents one of the most controversial issues in cc RCC. Resistance to therapy is based on an incomplete mechanism of action (21), and there is no effective definitive therapeutic tool available (22). Lichner et al. recently reported that Sunitinib therapy for advanced RCC induces selection of a tumor cells subpopulation able to survive and to grow as spheroids in tumor models xenografts (23). The group also showed that these cells express E cadherin as a key contributor to the survival of RCC cells under Sunitinib treatment. The Memorial Sloan Kettering Cancer Centre RCC classification (Motzer criteria) focuses on clinical features of RCC to predict survival. This system evaluates: i) performance status, ii) LDH value, iii) haemoglobin value, iv) serum calcium concentration and v) time from initial diagnosis to systemic treatment (24); however, no pathological variables are taken into consideration. A previous attempt to integrate the clinical data with tissue markers so as to predict disease mortality in metastatic RCC was done by Kim et al. (25), which, unfortunately, highlighted the limitations. Taken together, it is clear that there is the strong need for novel prognostic biological markers reliably assessing tumor invasion as well as its metastatic potential in cc RCC. These tools are also needed for the identification of patients at higher risk of recurrence following local treatment.

CLIC1 is expressed in the normal kidney in the glomerular structures and also on the apical domain of proximal tubules' epithelial cells (26), however, its role of in renal cancer is yet to be elucidated. The Human Atlas Gene Protein mentions CLIC1 as an unfavorable prognostic marker in renal cancer, with no adequate details available on it (https://www. proteinatlas.org/ENSG00000213719-CLIC1/t).

Evidence regarding the interrelation and crosstalk between CLIC1 and RCC tumor cells derive mostly from in vitro and experimental tumor xenograft studies using different renal cancer cell lines or tumor cells isolated from patients with localized and metastatic RCC (15). CLIC1 inhibition seems to decrease tumor invasion and metastasis due to a blockage of the myosin light chain kinase (MYLK) and of $\beta 3$ integrin, suggesting an important role for CLIC1 in integrin-mediated actomyosin dynamics in cells with invasive and metastatic potential.

Despite of clear experimental evidence about the role of CLIC1 in RCC progression, no data are available about its expression on human cc RCC specimens at present. Thus, we aimed to describe CLIC1 expression pattern using immunohistochemistry on human tumor samples of cc RCC and to correlate the pattern of reaction with tumor grade, invasion and tumor heterogeneity.

\section{Materials and Methods}

Tumor samples. Fifty cases of cc RCC were collected during open surgery performed for kidney tumor mass of suspicious or proven malignant origin. Initial diagnosis was made by imaging (e.g. CT scan, MRI). Both localized and metastatic diseases were studied. Informed consent was obtained from all patients before surgery and all procedures respected ethical principles regarding the use of human tissue specimens for research purposes, according with WMA Declaration of Helsinki (https://www.wma.net/policiespost/wma-declaration-of-helsinki-ethical-principles-for-medicalresearch-involving-human-subjects/).

Tissue primary processing. Tumor samples fixed for $24 \mathrm{~h}$ in $10 \%$ buffered formalin were included in a routine automated workflow of paraffin embedding by using automated ThermoShandon carousel for histopathology (ThermoScientific Fischer, Cambridge, UK) including detailed protocols for each type of tissue. Automatic workflow follows the routine steps widely known in histopathology and has as a final product the parraffin block ready for sectioning Three micrometer-thick serial sections were cut from each specimen and were stained with haematoxylin and eosin for histopathological evaluation.

Immunohistochemistry. Following initial evaluation, additional paraffin-embedded slides from each RCC case were immunostained using the monoclonal mouse anti human CLIC1 antibody (Clone 356.1, dilution 1:2,000) (Santa Cruz Biotechnology, Heidelberg, Germany), incubated at room temperature for $30 \mathrm{~min}$. Incubation with the primary antibody was followed by the use of Bond Polymer Refine Detection System (Leica Biosystems, Newcastle Upon Tyne, UK) specific for BOND MAX autostainer having well standardized protocol including all steps of routine immunohistochemistry. Automated process selected into BOND MAX Autostainer performs automated dewax for $30 \mathrm{~min}$, incubation with primary antibodies as it was described before, and then incubation with polymer for $30 \mathrm{~min}$ followed by DAB chromogen for $10 \mathrm{~min}$. Counterstain was the final step automated immunostaining. Prostate tissue expressing CLIC1 was used as external positive control, and normal kidney tissue adjacent to the tumor as internal positive control. A dark brown color detected by microscopy on stained specimens having nuclear, cytoplasmic and/or membranar pattern was considered as positive for CLIC1. All immunohistochemical steps were fully automated and controlled by the Bond Max autostainer (Leica Biosystems, 


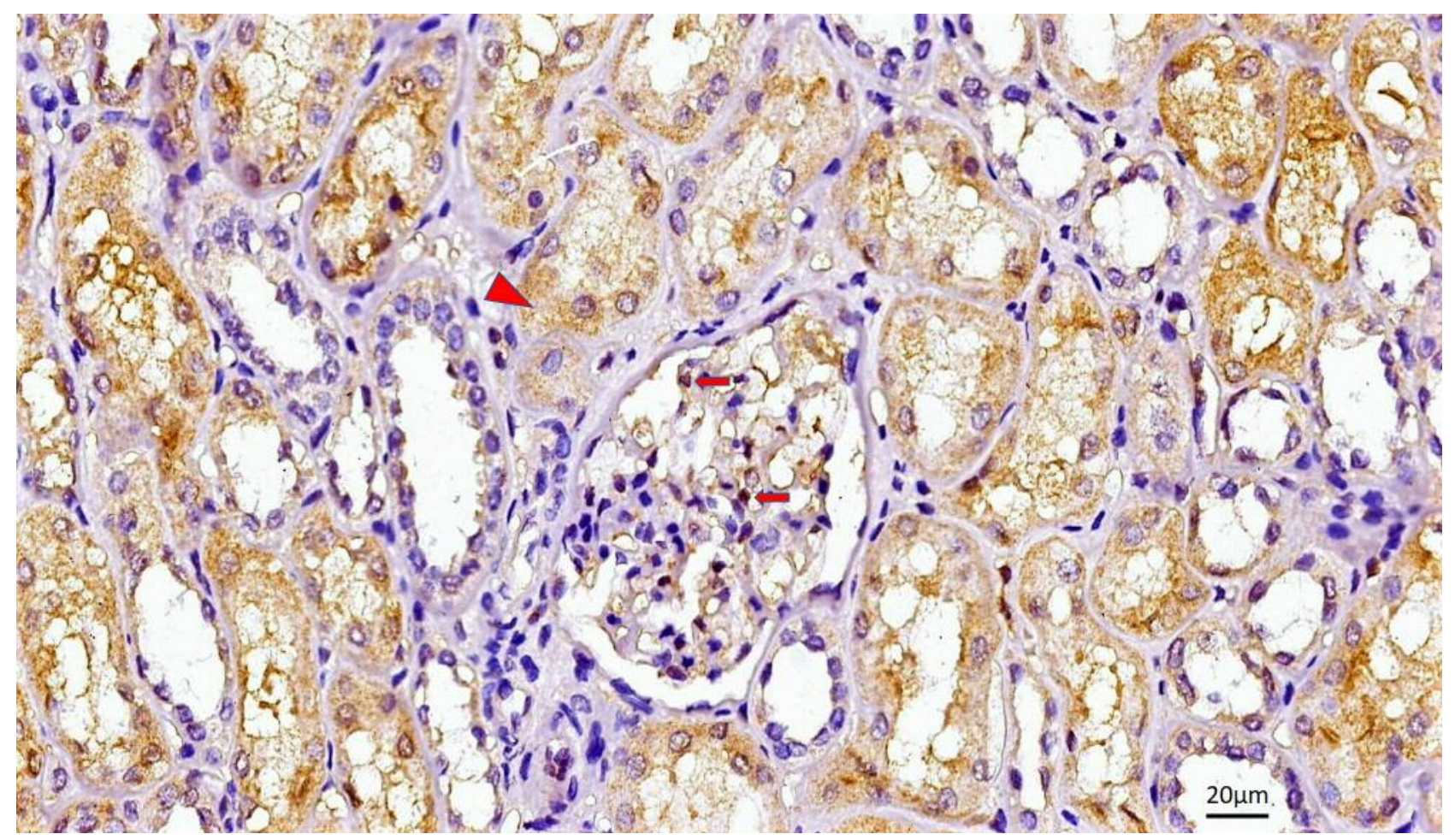

Figure 1. CLIC1 expression in the normal human renal parenchyma. Note the CLIC1 nuclear expression inside renal corpuscules (red arrow, podocytes) and its cytoplasmic expression in the proximal renal tubules (red arrowhead). Immunohistochemistry for CLIC1, x400 magnification.

Newcastle Upon Tyne, UK). The interpretation of immunostained specimens included all three patterns: i) nuclear $(\mathrm{N})$, ii) cytoplasmic (C) and iii) membrane (M), but we also checked combined patterns: i) all three (NMC), ii) nuclear and cytoplasmic (NC), iii) nuclear and membranar (NM), iv) cytoplasmic and membrane (CM). We stratified cases according to the ammounts of CLIC1 positive cells as follows: i) low expressing tumors (10-30\% of tumor cells expressed CLIC1), ii) mild expressing tumors (30-50\%) and iii) high expressing tumors ( $>50 \%$ tumor cells positive for CLIC1). The intensity of positive reaction was then assessed as: i) weak (1), ii) moderate (2) and iii) strong (3).

Image aquisition and data analysis. All slides were scanned using the Pannoramic Desk slide scanner (3D Histech, Budapest, Hungary). Digital slides were stored in Case Center and were assessed by using the Pannoramic Viewer Platform (3D Histech, Budapest, Hungary). By using these methods we evaluated the whole section of each specimen for CLIC1 expression pattern, percentage of positive cells and CLIC1 signal intensity. Statistical analysis was performedusing SPSS version 17 , for evaluating correlations tests (Pearson, Spearman and Kendall). A $p$-value less than 0.05 being considered statistically significant.

\section{Results}

The normal kidney tissue adjacent to the tumor presented a heterogeneously positive reaction for CLIC1. The renal cortex was intensely stained compared to the medulla. We persistently detected CLIC1 expression inside the renal corpuscle with a nuclear pattern restricted to podocytes. The number of positive podocytes was significantly lower compared to the presumed total number of podocytes in the normal glomerulus. The tubular system of the normal kidney had a different expression, the strongest immunostaining was observed inside the proximal renal tubules compared to distal and collecting tubes where a weak CLIC1 expression or negative reaction was found (Figure 1).

Within the tumor tissue, CLIC1 was expressed in malignant cells and in the endothelium of small intratumoral and peritumoral blood vessels. CLIC1 showed high accuracy in detecting locoregional and intravascular invasion thanks to its high ability of intensively staining tumor cells, unlike the staining of the surrounding tissues. Endothelial cells lining some tumor capillaries expressed CLIC1 as well.

CLIC1 was detected in $87.5 \%$ of all the examined cases of cc RCC. Positive reaction was homogeneously distributed in the whole tumor area in $31.42 \%$ of the total positive cases. For the remaining cases (68.57\%), positive tumor cells were distributed in a "mosaic-like" fashion, with groups of positive tumor cells intermingled within negative areas (Figures 2A and B). 53.33\% out of G2 cases and $91.66 \%$ of G3 cases had a heterogeneous distribution of CLIC1 positivity. Heterogeneity of expression increased with tumor 


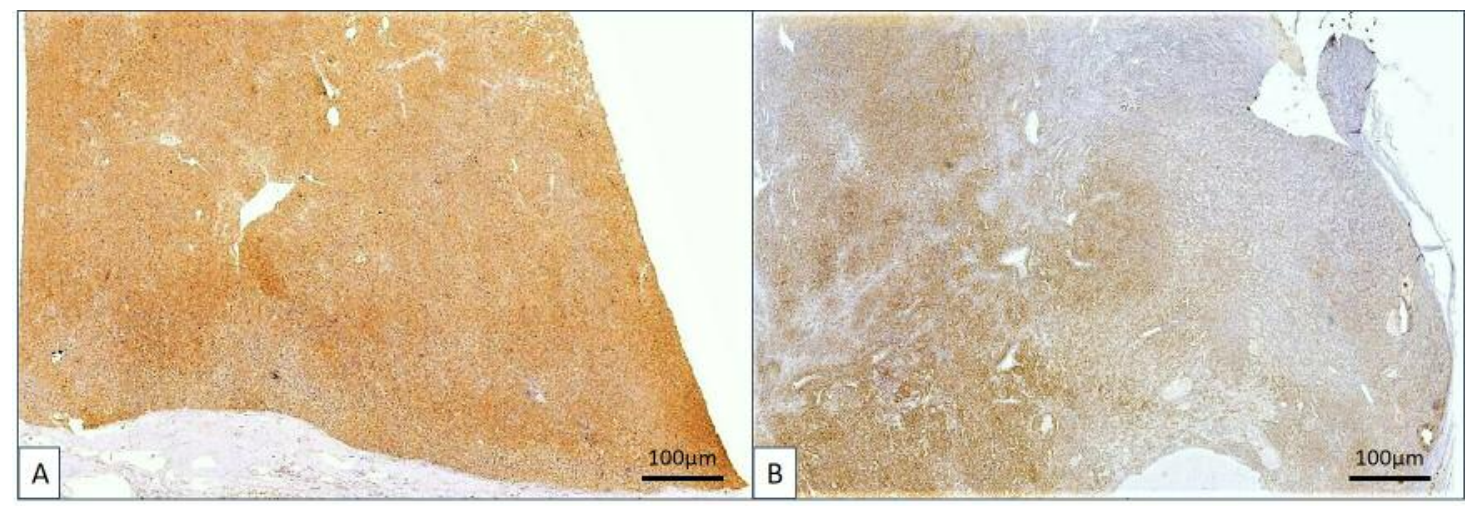

Figure 2. Homogeneous (A) versus heterogeneous (B) distribution of CLIC1 immunopositive reaction inside cc RCC (CLIC1 immunohistochemistry, $\times 40$ magnification).

grading, the most heterogeneous distribution of positive reaction for CLIC1 observed for G3 tumors (Figure 3). Based on this distribution pattern, we thought appropiate to quantify the percent of positive cells from each case and we found that $8.57 \%$ of all positive cases were CLIC1 lowexpressing tumors, $17.14 \%$ were CLIC1 mild-expression tumors and $71.42 \%$ were CLIC1 high-expressing tumors. The WHO/ISUP grading system was used to assess nuclear and nucleolar features; in this classification, tumor grade is assigned according to the highest grade cells present in the tissue analyzed (27).

Following an initial evaluation regarding CLIC1 positivity/negativity in cc RCC, we distributed cases depending on CLIC1 presence into i) nucleus (N), ii) cytoplasm (C) and iii) membrane (M) of tumor cells. Few cases had pure $\mathrm{N}$, M, or C location of CLIC1 positive expression, most of them displayed a combined pattern. Thus, we defined 7 subgroups of cc RCC, three with single expression $\mathrm{N}, \mathrm{M}$ or $\mathrm{C}$ and four with a combined expression as follows: iv) nuclear and cytoplasmic (NC), v) nuclear and membrane (NM), vi) cytoplasmic and membrane (CM), and vii) nuclear/membrane/cytoplasmic (NMC) (Figure 4).

For G3 tumors, CLIC1 cytoplasmic expression was strongly correlated with CLIC1 high-expressing tumors $(p=0.025)$ and also with tumor heterogeneity $(p=0.004)$. No G4 tumors were identified in the cases evaluated.

By analyzing our results we observed a high and dynamic heterogeneity of seven patterns related to tumor grade and CLIC1 expressing pattern. We also observed that in tumours with strong expression of CLIC1, the pattern of heterogeneity significantly increased depending on G. Pure N pattern (without any combination) was found only in CLIC1-high expressing group, for G2 tumors. Pure C pattern was persistently expressed for low-, mild- and high-subclasses, but it gradually increased in high-expressing tumors. Another important observation was that in the low-expressing group the $\mathrm{C}$ pattern was related to $\mathrm{G} 2$, while in the high-expressing group it was related to G3. Pure M pattern was observed only in CLIC1-high expressing tumors related to G2.

Assessement of combined patterns revealed that the NC pattern was present in all three expressing groups, however, it was most prominent in the high-expressing group.

We found particular dynamics in the NM and CM patterns within the group of high CLIC1-expressing tumors. NM pattern gradually decreased from G1 to G3 tumors, while $\mathrm{CM}$ pattern increased for $\mathrm{G} 2$ tumors compared to G1. Inside G3 subgroup already containing NM and CM patterns it was also observed the expression of NMC pattern which was not observed in G1 and G2 subgroups of tumors with high CLIC1 expression.

Based on these evidences together with significant correlations found between the parameters assessed in the present study we may define four risk groups of cc RCC depending on G, CLIC1 expression and pattern: i) G3/NM/low CLIC1 expression (1), ii) G2/CM/mild CLIC1 expression (2), iii) $\mathrm{G} 1$ or G2 /NM or CM /high CLIC1 expression (3) and iv) G2/M /high CLIC1 expression (4) (Figure 5).

Statistically significant correlations between the different parameters used in the present study are summarized in Table I.

We also assessed the CLIC1 expression in relation to TNM classification parameters. For cc RCC having a combined nuclear and cytoplasmic CLIC1 expression, $\mathrm{M}$ parameter was significantly correlated with G2 $(p=0.045)$ while $\mathrm{N}$ parameter showed a strong correlation with percentage of CLIC1 positive tumor cells $(p=0.008)$. For concomitant nuclear and membranar CLIC1 expressing tumors, we found that $\mathrm{N}$ parameter had three significant correlations with $\mathrm{G}(p=0.039)$, the heterogeneity in CLIC1 expression $(p=0.031)$ and the percentage of positive tumor cells $(p=0.026)$. An interesting difference has been found between males and females regarding TNM parameters 


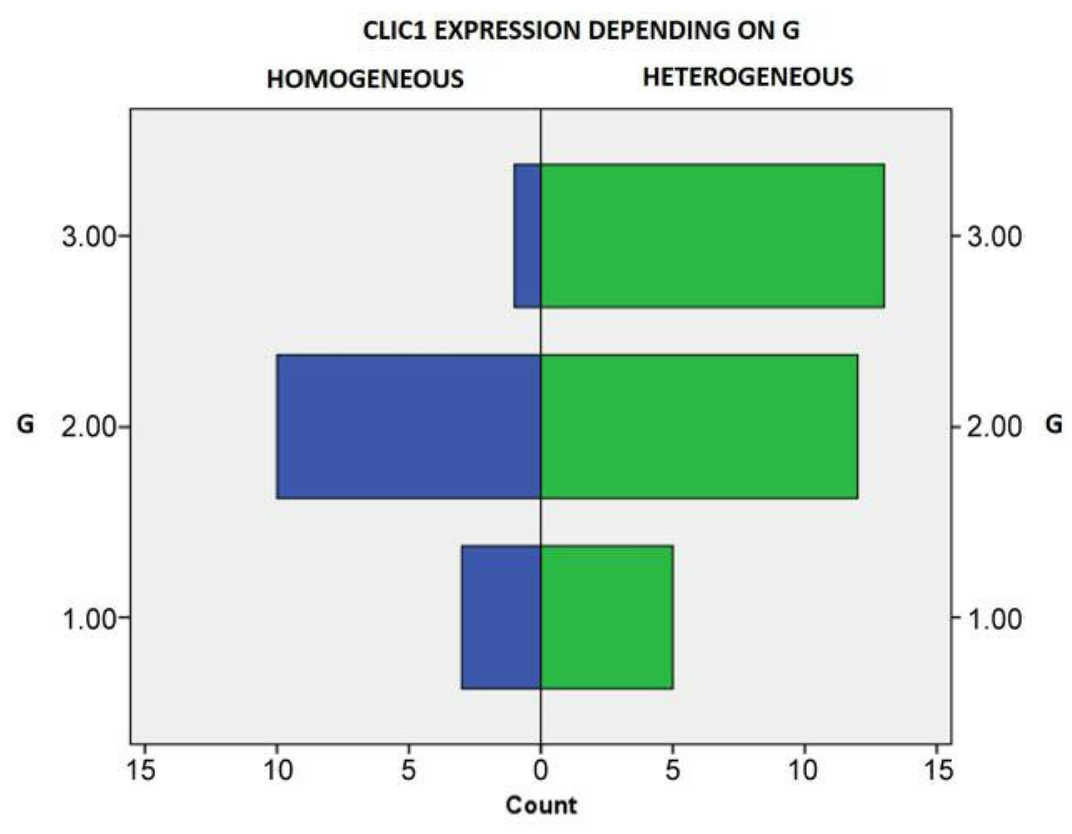

Figure 3. CLIC1 heterogeneity expression according to tumor grade.

associated with CLIC1 expression. In males, T parameter seems to significantly depend on the CLIC1 expression pattern $(p=0.014)$ and $\mathrm{N}$ parameter on the percentage of positive tumor cells $(p=0.043)$. No significant correlations of TNM with other parameters used in the present study were identified in the women's group.

\section{Discussion}

CLIC1 is known as ,sensor and effector” of different cellular processes involving the overproduction of reactive oxygen species (ROS) in several benign $(28,29)$ and malignant conditions $(10,11)$. Among malignant tumors, renal cell carcinomas are well known as being highly ROS producing tumors and this is strongly correlated with high tumor grade, advance stage and metastatic behaviour (30). Based on these data of high ROS production by RCC, already stated in the literature and related with our results about CLIC1 expression, we may speculate in this moment that the high expression of CLIC1 certified here by our results may acts as sensor or effector of cellular proliferation, invasion and metastasis. Also, there is some experimental evidence supporting oxidative stress inducing epigenetic changes in malignant renal cell lines (31). Despite this evidence, the role of CLIC1 expression in cc RCC has not been reported in the literature before.

The present study examines for the first time the CLIC1 expression in cc RCC. Surprisingly, a high number of cc RCC cases from our cohort showed CLIC1 positivity not only in the malignant cells of the primary tumor, but also in the neoplastic cells found in the surrounding connective tissue or in blood vessels. It might be that the pattern of CLIC expression is a useful tool for a better assessement of local and vascular invasion in cc RCC, as it is able to detect, not only groups of invasive cells, but also isolated cells spreading around the tumor or invading tumor-associated blood vessels.

The fact that CLIC1 is expressed in the nucleus, cytoplasm and/or membrane of tumor cells suggests different possible biological functions, depending on its location. Usually, CLIC1 has a cytoplasmic localization in normal human tissue and it translocates to the plasma membrane during malignant transformation (32). Setti et al. have proved that CLIC1 integration into the plasma membrane of tumor cells induces a high tumorigenic potential of glioblastoma cells. Also, CLIC1 is constitutively expressed in the plasma membrane of stem/progenitor cells (32). Pure $\mathrm{M}$ pattern appeared in our study only for ccRCC tumors from high-CLIC1-expressing group with G2. Highexpressing group showed the highest heterogeneity regarding the CLIC1 expression pattern, however, most of them were defined by NM or CM pattern. A special attention should be paid to those subgroups containing the M pattern. According with Peretti et al., the fact that CLIC1 membrane expression is crucial for cancer stem cells proliferation it may represent a potential therapeutic target (14). In accordacne with this, cc RCC subgroups with M pattern may be considered of high risk. Our results support the existence of several cc RCC subgroups, but the data are not yet sufficient to prove whether CLIC1 overexpression may be considered as a 


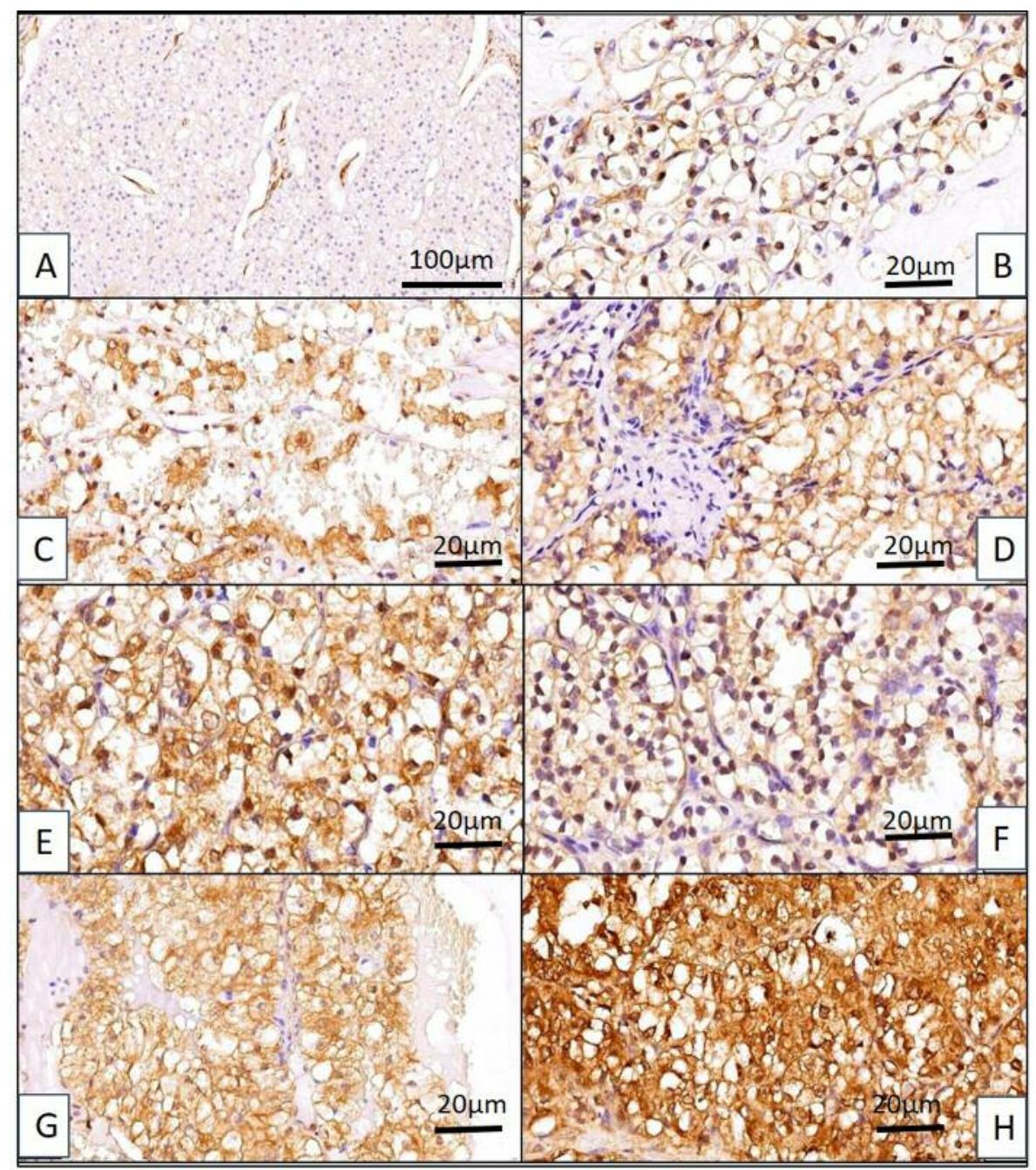

Figure 4. Seven expression patterns of CLIC1 have been identified in ccRCC. (A) CLIC1-negative ccRCC (note that tumor blood vessels are CLIC1 positive). (B) Nuclear pattern $(N),(C)$ cytoplasmic pattern $(C),(D)$ membrane pattern $(M),(E)$ nuclear/cytoplamic pattern $(N C)$, $(F)$ nuclear membrane $(N M),(G)$ cytoplasmic/membrane $(C M)$ and $(H)$ nuclear/ cytoplasmic/membrane pattern (NMC).

marker of aggresiveness. Further research should better define these aspects in cc RCC, and assess whether there is a possible role for CLIC1 as a marker for tumor risk stratification.

Another important aspect of CLIC1 role is its possibile involvement with tumor invasion and metastasis. We observed in our study a strong CLIC1 expression in cc RCC cancer cells invading surrounding tissues as well as in cells detected in the lumen of tumor-associated blood vessels. Moreover, focal areas of the strongest expression levels of CLIC1 were also detected at the periphery of several cc RCC. Gurski et al. have conducted the only study regarding CLIC1 expression in cancer cells derived from patients with metastatic and localized kidney cancer (15). They proved that CLIC1 translocation into the membrane acts as an activator of invadopodia development and sustains metastatic potential of cancer cells derived from kidney cancer. The same team demonstrated that a similar mechanism may act also in endothelial cells and promotes new blood vessel formation. In our study we also observed several intratumoral and peritumoral small blood vessels expressing CLIC1. This aspect may be due to exosome-based exchange between tumor cells and tumor microenvironment cells, such as endothelial cells (33). Such a crosstalk could provide a route responsible for vascular invasion and metastasis. Recently, indeed, Thuringer et al. have reported this very crosstalk between glioblastoma cells and endothelial cells that is mediated by CLIC1 released through extracellular vesicles by tumor cells and captured by endothelial cells, which are induced to sprout and form tubes in 3D matrices (34). 


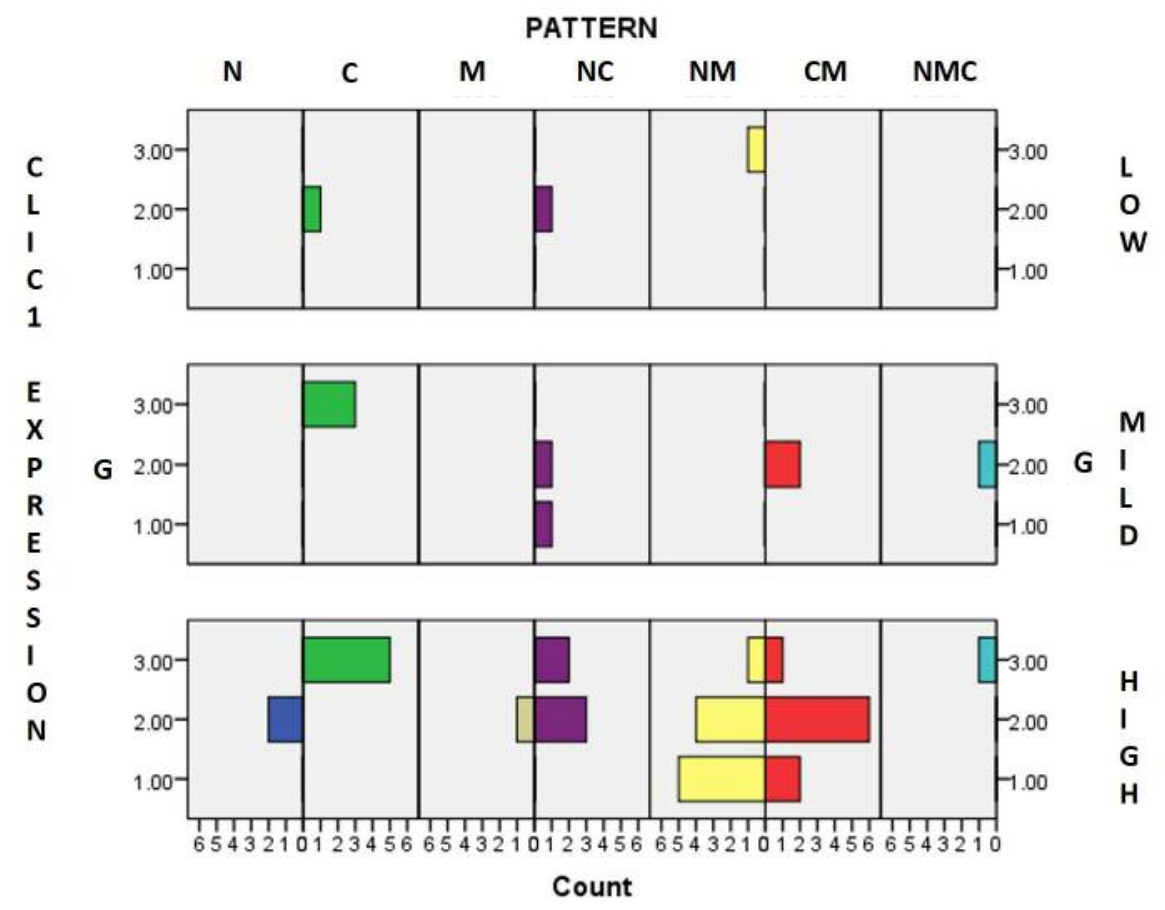

Figure 5. Cc RCC subgroups according to tumor grade (G), CLIC1 low, mild or strong expression and pattern as nuclear (N), cytoplasmic (C), membranar $(M)$, nuclear and cytoplasmic (NC), nuclear and membrane (NM), cytoplasmic and membrane (CM), and nuclear/membrane/cytoplasmic (NMC).

Table I. Correlations between CLIC1 parameters and tumor grade. All values highlighted in yellow support our results regarding the influence of CLIC1 expression on tumor grade $(G)$ and pattern heterogeneity.

\begin{tabular}{|c|c|c|c|c|c|c|}
\hline & & G & HET & Pattern & Positivity & Intensity \\
\hline \multirow[t]{3}{*}{ G } & Pearson's Correlation & 1 & $0.337 *$ & $-0.348^{*}$ & -0.133 & $-0.344 *$ \\
\hline & Sig. (2-tailed) & & 0.017 & 0.013 & 0.358 & 0.014 \\
\hline & $\mathrm{N}$ & 50 & 50 & 50 & 50 & 50 \\
\hline \multirow[t]{3}{*}{ HET } & Pearson's Correlation & $0.337 *$ & 1 & -0.182 & -0.198 & $-0.566^{* *}$ \\
\hline & Sig. (2-tailed) & 0.017 & & 0.206 & 0.167 & 0.000 \\
\hline & $\mathrm{N}$ & 50 & 50 & 50 & 50 & 50 \\
\hline \multirow[t]{3}{*}{ Pattern } & Pearson's Correlation & $-0.348^{*}$ & -0.182 & 1 & 0.107 & $0.437 * *$ \\
\hline & Sig. (2-tailed) & 0.013 & 0.206 & & 0.459 & 0.001 \\
\hline & $\mathrm{N}$ & 50 & 50 & 50 & 50 & 50 \\
\hline \multirow[t]{3}{*}{ Positivity } & Pearson's Correlation & -0.133 & -0.198 & 0.107 & 1 & $0.457 * *$ \\
\hline & Sig. (2-tailed) & 0.358 & 0.167 & 0.459 & & 0.001 \\
\hline & $\mathrm{N}$ & 50 & 50 & 50 & 50 & 50 \\
\hline \multirow[t]{3}{*}{ Intensity } & Pearson's Correlation & $-0.344 *$ & $-0.566 * *$ & $0.437 * *$ & $0.457 * *$ & 1 \\
\hline & Sig. (2-tailed) & 0.014 & 0.000 & 0.001 & 0.001 & \\
\hline & $\mathrm{N}$ & 50 & 50 & 50 & 50 & 50 \\
\hline
\end{tabular}

*Correlation is significant at the 0.05 level (2-tailed). ${ }^{* *}$ Correlation is significant at the 0.01 level (2-tailed).

CLIC1 has already been reported as a potential biomarker for some malignant tumors $(6,10,16)$ and a potential therapeutic target for new treatments in cancer. Its expression in both tumor and endothelial cells may pave the way for the development of future anti-CLIC1 therapy, able to target both tumor and its associated vessels.
There are some data available concerning the inhibitory effects of metformin (a well-known oral antidiabetic drug, recently and intensely spotlighted as having anti-cancer effects by suppressing oncogenic signaling pathways, including receptor tyrosine kinase, PI3K/Akt, and mTOR pathways) on CLIC1 expression in malignant tumors, especially against 
cancer stem cells (35). Among all the different mechanisms proposed for metformin-mediated antiproliferative activity there is also the inhibition of CLIC1 activity. Gastric cancer cell lines SGC-7901 and MGC-803 express CLIC1 (36). The specific inhibitor indanyloxyacetic acid-94 (IAA94) prevents CLIC1 from translocating in the cell membrane and through this action it prevents cell aggregation and proliferation (37). Silencing of the CLIC1 promotes apoptosis and decreases gallbladder cancer cells proliferation and migration (38).

In this study we report, for the first time, the expression and histopathological significance of CLIC1 in human cc RCC specimens, as detected by immunohistochemistry. CLIC1 expression stratified cc RCC in four subgroups depending on tumor grade, CLIC1 expression pattern and percent of tumor-positive cells. Further investigations are needed to elucidate the prognostic and therapeutic impact of CLIClin cc RCC.

\section{Conflicts of Interest}

The Authors have no conflict of interests to declare.

\section{Authors' Contributions}

AN wrote the paper and participated into the study. MR designed the study. MR and AMC evaluated histopathology and immunohistochemistry of the specimens, revised the first draft of the manuscript. AA and II are surgeons participating to tumor removal. TCC, CP and $\mathrm{MM}$ provided the antibody and participated to the manuscript writing.

\section{Acknowledgements}

The Authors are grateful for the financial support provided by Victor Babes University of Medicine and Pharmacy Timisoara, from its internal research funds during implementation of the project RCC AMIRA, pIII-C4-PCFI-2016/2017-01. They would also like to thank the histotechnologists Ciprian Onica and Patricia Berzava for their excellent technical support.

\section{References}

1 Wang W, Xu X, Wang W, Shao W, Li L, Yin W, Xiu L, Mo M, Zhao J, He Q and He J: The expression and clinical significance of CLIC1 and HSP27 in lung adenocarcinoma. Tumour Biol 32: 1199-1208, 2011. PMID: 21858536. DOI: 10.1007/s13277-0110223-0

2 Littler DR, Harrop SJ, Fairlie WD, Brown LJ, Pankhurst GJ, Pankhurst S, DeMaere MZ, Campbell TJ, Bauskin AR, Tonini R, Mazzanti M, Breit SN and Curmi PM: The intracellular chloride ion channel protein CLIC1 undergoes a redoxcontrolled structural transition. J Biol Chem 279: 9298-9305, 2004. PMID: 14613939. DOI: 10.1074/jbc.M308444200

3 Goodchild SC, Howell MW, Cordina NM, Littler DR, Breit SN, Curmi PM and Brown LJ: Oxidation promotes insertion of the CLIC1 chloride intracellular channel into the membrane. Eur Biophys J 39(1): 129-138, 2009. PMID: 19387633. DOI: 10.1007/s00249-009-0450-0
4 Peretti M, Angelini M, Savalli N, Florio T, Yuspa SH and Mazzanti M: Chloride channels in cancer: Focus on chloride intracellular channel 1 and 4 (CLIC1 AND CLIC4) proteins in tumor development and as novel therapeutic targets. Biochim Biophys Acta 1848: 2523-2531, 2015. PMID: 25546839. DOI: 10.1016/j.bbamem.2014.12.012

5 Li BP, Mao YT, Wang Z, Chen YY, Wang Y, Zhai CY, Shi B, Liu SY, Liu JL and Chen JQ: CLIC1 Promotes the Progression of Gastric Cancer by Regulating the MAPK/AKT Pathways. Cell Physiol Biochem 46: 907-924, 2018. PMID: 29669336. DOI: $10.1159 / 000488822$

$6 \mathrm{Xu} \mathrm{Y,} \mathrm{Xu} \mathrm{J,} \mathrm{Feng} \mathrm{J,} \mathrm{Li} \mathrm{J,} \mathrm{Jiang} \mathrm{C,} \mathrm{Li} \mathrm{X,} \mathrm{Zou} \mathrm{S,} \mathrm{Wang} \mathrm{Q} \mathrm{and} \mathrm{Li}$ Y: Expression of CLIC1 as a potential biomarker for oral squamous cell carcinoma: a preliminary study. Onco Targets Ther 11: 8073-8081, 2018. PMID: 30519049. DOI: 10.2147/ OTT.S181936

7 Setti M, Osti D and Richichi C: Extracellular vesicle-mediated transfer of CLIC1 protein is a novel mechanism for the regulation of glioblastoma growth. Oncotarget 6: 31413-31427, 2015. PMID: 26429879. DOI: $10.18632 /$ oncotarget.5105

8 Barbieri F, Würth R, Pattarozzi A, Verduci I, Mazzola C, Cattaneo M G, Tonelli M, Solari A, Bajetto A, Daga A, Vicentini L M, Mazzanti $M$ and Florio $T$ : Inhibition of chloride intracellular channel 1 (CLIC1) as biguanide class-effect to impair human glioblastoma stem cell viability. Front Pharmacol 9: 899, 2018. PMID: 30186163. DOI: 10.3389/fphar.2018. 00899.

9 Lu J, Dong Q, Zhang B, Wang X, Ye B, Zhang F, Song X, Gao $\mathrm{G}, \mathrm{Mu}$ J, Wang Z, Ma F and Gu J: Chloride intracellular channel 1 (CLIC1) is activated and functions as an oncogene in pancreatic cancer. Med Oncol 32: 616, 2015. PMID: 25920608. DOI: $10.1007 / \mathrm{s} 12032-015-0616-9$

10 Singha B, Harper SL, Goldman AR, Bitler BG, Aird KM, Borowsky ME, Cadungog MG, Liu Q, Zhang R, Jean S, Drapkin R and Speicher DW: CLIC1 and CLIC4 complement CA125 as a diagnostic biomarker panel for all subtypes of epithelial ovarian cancer. Sci Rep 8: 14725, 2018. PMID: 30282979. DOI: 10.1038/s41598-018-32885-2

11 Kobayashi T, Shiozaki A, Nako Y, Ichikawa D, Kosuga T, Shoda K, Arita T, Konishi, H, Komatsu S, Kubota T, Fujiwara H, Okamoto K, Kishimoto M, Konishi E, Marunaka Y and Otsuji E: Chloride intracellular channel 1 as a switch among tumor behaviors in human esophageal squamous cell carcinoma. Oncotarget 9: 23237-23252, 2018. PMID: 29796185 PMCID: 29796185. DOI: 10.18632/oncotarget.25296

12 Liao PC and Chang YH: CLIC1 (chloride intracellular channel 1). Atlas of genetics and cytogenetics in oncology and haematology 16: 266-269, 2011.

13 Hossain KR, Holt SA, Le Brun AP, Al Khamici H and Valenzuela SM: X-ray and neutron reflectivity study shows that CLIC1 undergoes cholesterol-dependent structural reorganization in lipid monolayers. Langmuir 33: 12497-12509, 2017. PMID: 29016141. DOI: 10.1021/acs.langmuir.7b02872

14 Peretti M, Raciti FM, Carlini V, Verduci I, Sertic S, Barozzi S, Garré M, Pattarozzi A, Daga A, Barbieri F, Costa A, Florio T and Mazzanti M: Mutual Influence of ROS, pH, and CLIC1 membrane protein in the regulation of G1-S phase progression in human glioblastoma stem cells. Mol Cancer Ther 17: 24512461, 2018. PMID: 30135216. DOI: 10.1158/1535-7163.MCT$17-1223$ 
15 Gurski LA, Knowles LM, Basse PH, Maranchie JK, Watkins SC and Pilch J: Relocation of CLIC1 promotes tumor cell invasion and colonization of fibrin. Mol Cancer Res 13: 273-280, 2014. PMID: 25205595. DOI: 10.1158/1541-7786.MCR-14-0249

16 Ye Y, Yin M, Huang B, Wang Y, Li X and Lou G: CLIC1 a novel biomarker of intraperitoneal metastasis in serous epithelial ovarian cancer. Tumour Biol 36: 4175-4179, 2015. PMID: 25582317. DOI: 10.1007/s13277-015-3052-8

17 Yuan H, Yan M, Zhang G, Liu W, Deng C, Liao G, Xu L, Luo T, Yan H, Long Z, Shi A, Zhao T, Xiao Y and Li X: CancerSEA: A cancer single-cell state atlas. Nucleic Acids Res 47: 900-908, 2018. PMID: 30329142 DOI: 10.1093/nar/gky939

18 Siegel RL, Mille KD and Jemal A: Cancer statistics, 2019. CA Cancer J Clin 69: 7-34, 2019. PMID: 30620402. DOI: $10.3322 /$ caac. 21551

19 Massari F, Di Nunno V, Mollica V, Graham J, Gatto L and Heng D: Adjuvant tyrosine kinase inhibitors in treatment of renal cell carcinoma: A meta-analysis of available clinical trials. Clin Genitourin Cancer 1558-7673: 30005-30009, 2019. PMID: 30704796. DOI: 10.1016/j.clgc.2018.12.011

20 Volpe A, Bollito E, Bozzola C, Di Domenico A, Bertolo R, Zegna L, Duregon E, Boldorini R, Porpiglia $F$ and Terrone C: Classification of histologic patterns of pseudocapsular invasion in organ-confined renal cell carcinoma. Clin Genitourin Cancer 14: 69-75, 2016. PMID: 26337654. DOI: 10.1016/j.clgc.2015. 07.020

21 Makhov P, Joshi S, Ghatalia P, Kutikov A, Uzzo RG and Kolenko VM: Resistance to systemic therapies in clear cell renal cell carcinoma: Mechanisms and management strategies. Mol Cancer Ther 17: 1355-1364, 2018. PMID: 29967214. DOI: 10.1158/1535-7163.MCT-17-1299

22 Duran I, Lambea J, Maroto P, González-Larriba JL, Flores L, Granados-Principal S, Graupera M, Sáez B, Vivancos A and Casanovas O: Resistance to targeted therapies in renal cancer: The importance of changing the mechanism of action. Target Oncol 12: 19-35, 2017. PMID: 27844272. DOI: 10.1007/s11523-016-0463-4.

23 Lichner Z, Saleeb R, Butz H, Ding Q, Nofech-Mozes R, Riad S, Farag M, Varkouhi AK, Dos Santos CC, Kapus A and Yousef GM: Sunitinib induces early histomolecular changes in a subset of renal cancer cells that contribute to resistance. FASEB J 33: 1347-1359, 2019. PMID: 30148679. DOI: 10.1096/fj.2018 00596R

24 Motzer RJ, Mazumdar M, Bacik J, Berg W, Amsterdam A and Ferrara J: Survival and prognostic stratification of 670 patients with advanced renal cell carcinoma. J Clin Oncol 17: 2530-2540, 1999. PMID: 10561319. DOI: 10.1200/JCO.1999.17.8.2530

25 Kim HL, Seligson D, Liu X, Janzen N, Bui MH, Yu H, Shi T, Belldegrun AS, Horvath S and Figlin RA: Using tumor markers to predict the survival of patients with metastatic renal cell carcinoma. J Urol 173: 1496-1501, 2005. PMID: 15821467. DOI: 10.1097/01.ju.0000154351.37249.f0

26 Tulk BM and Edwards JC: NCC27, a homolog of intracellular Cl- channel p64, is expressed in brush border of renal proximal tubule. Am J Physiol 274: 1140-1149, 1998. PMID: 9841507. DOI: 10.1152/ajprenal.1998.274.6.F1140

27 Delahunt B, Cheville JC, Martignoni G, Humphrey PA, MagiGalluzzi $\mathrm{C}$ and McKenney J: The International Society of Urological Pathology (ISUP) grading system for renal cell carcinoma and other prognostic parameters. Am J Surg Pathol 37: 1490-1504, 2013. PMID: 24025520. DOI: 10.1097/ PAS.0b013e318299fofb
28 Averaimo S, Milton RH, Duchen MR and Mazzanti M: Chloride intracellular channel 1 (CLIC1): Sensor and effector during oxidative stress. FEBS Lett 584: 2076-2084, 2010. PMID: 20385134. DOI: 10.1016/j.febslet.2010.02.073

29 Liu B, Billington C K, Henry A P, Bhaker S K, Kheirallah A K, Swan C and Hall IP: Chloride intracellular channel 1 (CLIC1) contributes to modulation of cyclic AMP-activated whole-cell chloride currents in human bronchial epithelial cells. Physiol Rep 6: 13508, 2018. PMID: 29368798. DOI: 10.14814/ phy2.13508

30 Ganesamoni R, Bhattacharyya S, Kumar S, Chauhan A, Mete UK, Agarwal MM, Mavuduru R, Kaushik G, Mandal AK and Singh SK: Status of oxidative stress in patients with renal cell carcinoma. J Urol 187: 1172-1176, 2012. PMID: 22335872. DOI: $10.1016 /$ j.juro.2011.11.105

31 Mahalingaiah P K, Ponnusamy L and Singh K P: Oxidative stress-induced epigenetic changes associated with malignant transformation of human kidney epithelial cells. Oncotarget 8 : 11127-11143, 2016. PMID: 27655674. DOI: 10.18632/ oncotarget.12091

32 Setti M, Savalli N, Osti D, Richichi C, Angelini M, Brescia P, Fornasari L, Carro M S, Mazzanti M and Pelicci G: Functional role of CLIC1 ion channel in glioblastoma-derived stem/ progenitor cells. J Natl Cancer Inst 105: 1644-1655, 2013. PMID: 24115360. DOI: 10.1093/jnci/djt278

33 Maia J, Caja S, Strano Moraes M C, Couto N and Costa-Silva B: Exosome-based cell-cell communication in the tumor microenvironment. Front Cell Dev Biol 6: 18, 2018. PMID: 29515996. DOI: $10.3389 /$ fcell.2018.00018

34 Thuringer D, Chanteloup G, Winckler P and Garrido C: The vesicular transfer of CLIC1 from glioblastoma to microvascular endothelial cells requires TRPM7. Oncotarget 9: 33302-33311, 2018. PMID: 30279961. DOI: 10.18632/oncotarget.26048

35 Gritti M, Würth R, Angelini M, Barbieri F, Peretti M, Pizzi E, Pattarozzi A, Carra E, Sirito R, Daga A, Curmi P M, Mazzanti $\mathrm{M}$ and Florio T: Metformin repositioning as antitumoral agent: selective antiproliferative effects in human glioblastoma stem cells, via inhibition of CLIC1-mediated ion current. Oncotarget 5: 11252-11268, 2014. PMID: 25361004. DOI: 10.18632/ oncotarget 2617

36 Ma P F, Chen J Q, Wang Z, Liu J L and Li B P: Function of chloride intracellular channel 1 in gastric cancer cells. World $\mathrm{J}$ Gastroenterol 18: 3070-3080, 2012. PMID: 22791942. DOI: 10.3748/wjg.v18.i24.3070

$37 \mathrm{Xu} \mathrm{Y,} \mathrm{Zhu} \mathrm{J,} \mathrm{Hu} \mathrm{X,} \mathrm{Wang} \mathrm{C,} \mathrm{Lu} \mathrm{D,} \mathrm{Gong} \mathrm{C,} \mathrm{Yang} \mathrm{J} \mathrm{and} \mathrm{Zong}$ L: CLIC1 inhibition attenuates vascular inflammation, oxidative stress, and endothelial injury. PLoS One 11: 0166790, 2016. PMID: 27861612. DOI: 10.1371/journal.pone.0166790

38 He Y M, Zhang Z L, Liu Q Y, Xiao Y S, Wei L, Xi C and Nan $\mathrm{X}$ : Effect of CLIC1 gene silencing on proliferation, migration, invasion and apoptosis of human gallbladder cancer cells. JCMM 22: 2569-2579, 2018. PMID: 29516682. DOI: 10.1111/ jemm.13499 